\title{
Evaluation method for behaviour of pipelines during earthquakes
}

\author{
K. Fuchida \\ Department of Civil \& Architectural Engineering, \\ Yatsushiro College of Technology, Kunamoto, Japan
}

\begin{abstract}
This paper presents a synthetic evaluating method for the seismic behaviour of pipelines during earthquakes. The proposed method consists mainly of three analytical methods, which are the reliability analysis method for lifeline networks, seismic response analysis of surface ground layers and the pseudo static response analysis of buried pipelines. The reliability analysis method for lifeline networks is based on the Monte Carlo method. The seismic response analysis method is two-dimensional effective stress analysis based on Biot's two-phase mixture theory and Iai's constitutive equation. The pipeline analysis is based on the beam theory on an elastic foundation and modified transfer matrix method. Numerical computations are performed for models with various conditions of ground and pipes. As a result, axial and lateral response characteristics of pipelines are shown and evaluated by relating with earthquakes, grounds and pipelines conditions.
\end{abstract}

Keywords: response analysis, pipeline, surface layers, reliability, network, cost.

\section{Introduction}

Severe damage to lifeline systems during earthquakes greatly affects the social activity and urban life of citizens. It is very important that aseismic investment for lifeline systems is performed in view of the fast recovery of economical and social works in urban areas after earthquakes.

This study aims to propose a synthetic evaluation method for pipelines constituting a network system during earthquakes. The proposed method consists of three analytical methods. From a perspective viewpoint, the whole network system is investigated and weak pipelines are extracted. Then, the responses of 
the surface ground layers, in which a weak pipeline is buried, are investigated. Finally, detailed responses of pipelines are evaluated based on input soil displacements, which are determined by referring to the above responses of the surface ground layers. The proposed method is briefly introduced as follows and some investigated examples of analyses are shown.

\section{Synthetic evaluation method for pipeline responses}

The synthetic method for evaluating pipeline responses during an earthquake is proposed here. This method consists of three main analytical methods. The first method is the reliability analysis for the network system in the object area $[1,2]$. The second method of the proposed synthetic method is the response analysis for the surface ground layers. The analysis is performed by the existing computer program, which is the effective stress analysis program "NUW2" [4, 5]. The program "NUW2" is based on Biot's two-phase mixture theory and Iai's constitutive equation [6]. The third method of the proposed synthetic method is the response analysis for the pipeline. This method is based on the beam theory on an elastic foundation and modified transfer matrix method $[8,9]$. The flow of this synthetic method is shown in Figure 1.

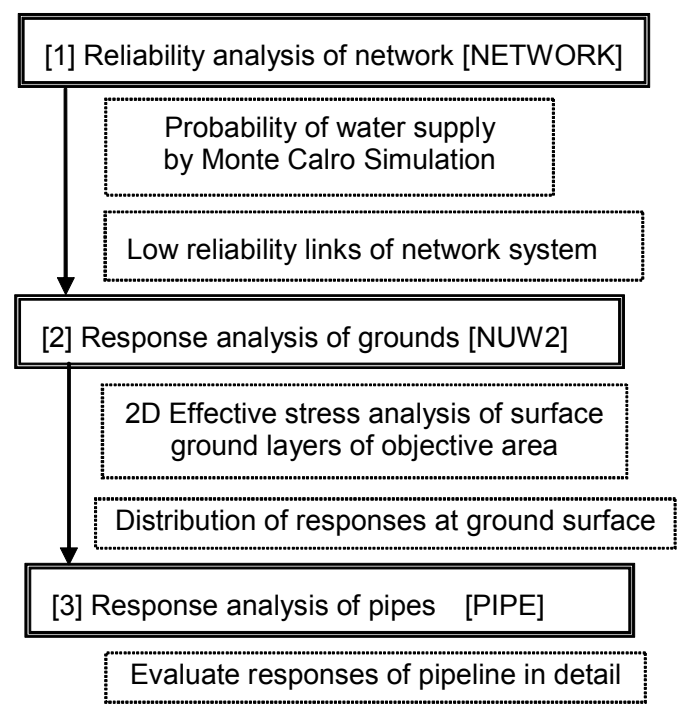

Figure 1: $\quad$ Flow of the synthetic analysis.

\subsection{Reliability analysis}

The flow of the reliability analysis is shown in Figure 2 [1]. In the first step, the network of pipelines is modelled into the node-link discrete system and the failure probability of each link is evaluated by Quantification theory I. The 
seismic intensity distribution for future earthquakes in the objective area is induced by using the past occurrence records of earthquakes in the respective area. The probability of the occurrence of earthquakes within a period is evaluated.

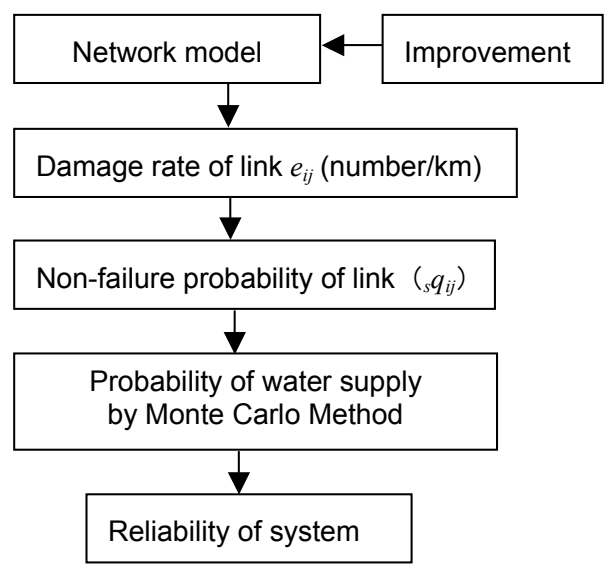

Figure 2: $\quad$ Flow of reliability analysis.

The failure of pipelines is dependent on the seismic intensity, the ground characteristics and the pipeline characteristics. By unifying the failure data of past earthquakes, which are Miyagi-ken-oki (1978), Nihonkai-chubu (1983), Kushiro-oki (1993) and Hyogo-ken-nanbu (1995), with Quantification theory I, the equation of regression for the failure ratio of the pipeline is derived in terms of the category weights. In this process the occurrence of liquefaction is estimated by the method of JRA [3].

In the second step of this analysis, the reliability for each link of a network system is computed by the Monte Carlo simulation technique [2], as is the corresponding failure probability, which is based on Quantification theory I, in terms of earthquake intensity, stiffness of the ground, the scale of liquefaction and the type of pipes. In this process, the connectivity of the system is defined as the rate of the existing path among the pair of nodes. The probability of system connectivity is also evaluated by the Monte Carlo simulation.

\subsection{Response analysis for surface ground}

The analytical method for the response of surface ground used in this study adopts two-dimensional dynamic equilibrium equations for the soil-water phase and generalized Darcy law for the pore water based on Biot's two-phase mixture theory as in $[4,5,7]$. To treat non-linearity (liquefaction) of the soil in the near field, the constitutive model for the plain condition is introduced. This constitutive model is constructed based on the 2-D strain-space multimechanism model for cyclic mobility of sandy soil, first proposed by Iai et al. [6]. For the far field condition, the absorbing boundary condition is prepared. 
The above dynamic equilibrium equations are formulated to the finite element equation by considering the irreducible weak Galerkin formulation. The matrix form of the finite element equation for a saturated porous medium with compressible pore water, including the absorbing boundary condition, may be written as:

$$
\begin{aligned}
& \left(\begin{array}{ll}
m_{u u} & m_{u w} \\
m_{u w}^{T} & m_{w w}
\end{array}\right)\left\{\begin{array}{c}
\ddot{\bar{u}} \\
\ddot{\bar{w}}
\end{array}\right\}+\left(\begin{array}{cc}
0 & 0 \\
0 & c_{w w}
\end{array}\right)\left\{\begin{array}{c}
\dot{\bar{u}} \\
\dot{\bar{w}}
\end{array}\right\}+\left(\begin{array}{cc}
K_{u u} & K_{u w} \\
K_{u w}^{T} & K_{w w}
\end{array}\right)\left\{\begin{array}{l}
\bar{u} \\
\bar{w}
\end{array}\right\}+\left\{\int_{\Omega} B_{u}^{T} \sigma^{\prime} d \Omega\right\} \\
& =\left\{\begin{array}{l}
\bar{f}_{u}+\bar{f}_{u}^{f} \\
\bar{f}_{w}+\bar{f}_{w}^{f}
\end{array}\right\}-\left(\begin{array}{cc}
c_{u u}^{\prime} & c^{\prime}{ }_{u w} \\
c_{u w}^{\prime T} & c^{\prime}{ }_{w w}
\end{array}\right)\left\{\begin{array}{l}
\dot{\bar{u}}-\dot{\bar{u}}^{f} \\
\dot{\bar{w}}-\dot{\bar{w}}^{f}
\end{array}\right\}
\end{aligned}
$$

where $\mathrm{u}$ and $\mathrm{w}$ are the nodal displacement vectors. Other notations can be seen in [5]. Equation (1) is coded as the 2D-FE effective stress analysis program of the name "NUW2".

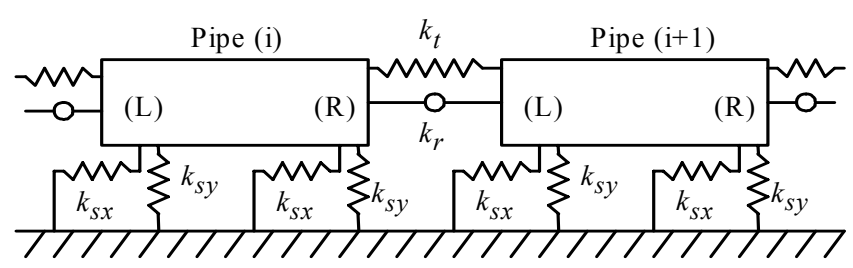

Figure 3: $\quad$ Modelling of pipeline-soil system.

\subsection{Response analysis for pipelines}

The responses of pipelines subjected to the ground displacement induced by liquefaction are analyzed by the program "PIPE" [8,9], which is based on the beam theory on an elastic foundation. Replacing the stiffness of liquefied ground around the pipeline with a coefficient of subgrade reaction, the pipeline is modelled as a pipeline-soil spring system as shown in Figure 3. The pipeline segments are connected by the axial and rotational joint springs $k_{\mathrm{t}}$ and $k_{r}$, respectively. The joint and soil springs are assumed to be bi-linearly elastic and the inertia and damping forces are neglected under the static load assumption. Based on these assumptions, when a ground motion is transmitted to a pipeline directly by a soil spring, the governing axial and lateral equations of the pipeline are written by

a) axial direction

$$
-E A \frac{d^{2} u}{d x^{2}}+k_{s x} u=k_{s x} u_{s}
$$

b) lateral direction

$$
E I \frac{d^{4} v}{d x^{4}}+k_{s y} v=k_{s y} v_{s}
$$

where $\mathrm{u}, \mathrm{v}=$ axial and lateral displacements of the pipeline respectively, $E, A$ and $I=$ Young's modulus, the cross sectional area and the geometrical moment of 
inertia of the pipeline respectively, $u_{\mathrm{s}}, v_{\mathrm{s}}=$ axial and lateral displacements of input ground motion respectively, $k_{\mathrm{sx}}, k_{\mathrm{sy}}=$ axial and lateral soil spring constants respectively. In this study the axial deformation of eq.(2) and the bending deformation of eq.(3) are uncoupled. For the prevention of numerical error accumulation, the modified transfer matrix method is adopted. According to this method, the vector $y$ for the displacements and the vector $z$ for the forces are correlated as follows:

$$
\begin{aligned}
& \vec{a} y+\vec{b} z=\vec{c} \\
& \bar{a} y+\overleftarrow{b} z=\vec{c}
\end{aligned}
$$

where $a, b$ are square matrices, and the symbol $\rightarrow, \leftarrow$ means the transfer from left to right and from right to left, respectively. Here, the state vector $V$ at the pipeline element is defined as

$$
V=[y, z]
$$

where $y=[u, v, \phi], z=[N, M, Q], \phi, N, M, Q=$ deflection angle, axial force, bending moment and shear force at the end of pipeline segment. Then, eqs.(2) and (3) are also represented by the system equation as

$$
\frac{d V}{d x}=A V+f(x)
$$

where

$$
A=\left[\begin{array}{cccccc}
0 & 0 & 0 & -1 / E A & 0 & 0 \\
0 & 0 & -1 & 0 & 0 & 0 \\
0 & 0 & 0 & 0 & -1 / E I & 0 \\
-k_{s x} & 0 & 0 & 0 & 0 & 0 \\
0 & 0 & 0 & 0 & 0 & 1 \\
0 & -k_{s y} & 0 & 0 & 0 & 0
\end{array}\right], f(x)=\left[\begin{array}{c}
0 \\
0 \\
q(x) \\
0 \\
0 \\
q(x)=k_{\mathrm{sx}} u_{\mathrm{s}}=\text { distributed axial load } \\
p(x)=k_{\mathrm{sy}} v_{\mathrm{s}}=\text { distributed lateral load }
\end{array}\right.
$$

A general solution of eq.(7) is given by

$$
V(x)=e^{A x} V(0)+e^{A x} \int_{0}^{x} e^{-A s} f(s) d s
$$

where $e^{A x}=$ transfer matrix.

The governing axial and lateral equations (2) and (3) are written the same as eq.(7);

$$
\begin{aligned}
& \frac{d}{d x}\left\{\begin{array}{l}
u \\
u^{\prime}
\end{array}\right\}=\left[\begin{array}{cc}
0 & 1 \\
\beta_{1}^{2} & 0
\end{array}\right]\left\{\begin{array}{l}
u \\
u^{\prime}
\end{array}\right\}+\left\{\begin{array}{l}
0 \\
-\beta_{1}^{2} u_{s}
\end{array}\right\} \\
& \frac{d}{d x}\left\{\begin{array}{c}
v \\
v^{\prime} \\
v^{\prime \prime} \\
v^{\prime \prime \prime}
\end{array}\right\}=\left[\begin{array}{cccc}
0 & 1 & 0 & 0 \\
0 & 0 & 1 & 0 \\
0 & 0 & 0 & 1 \\
-4 \beta_{2}^{4} & 0 & 0 & 0
\end{array}\right]\left\{\begin{array}{c}
v \\
v^{\prime} \\
v^{\prime \prime} \\
v^{\prime \prime \prime}
\end{array}\right\}+\left\{\begin{array}{c}
0 \\
0 \\
0 \\
4 \beta_{2}^{4} v_{s}
\end{array}\right\}
\end{aligned}
$$

where $\beta_{1}=\sqrt{k_{s x} / E A}, \beta_{2}=\sqrt[4]{k_{s x} / E I}$, means the differential $d / d x$.

Using the vector $V$, the field transfer matrix $e^{A x}$ can be obtained. At the joint between the pipe segments $\mathrm{i}$ and $\mathrm{i}+1$, the following relations are obtained by considering the conditions of displacement and equilibrium; 


$$
\begin{aligned}
& u_{i+1}^{L}=u_{i}^{R}-N_{i}^{R} / k_{T}, \quad v_{i+1}^{L}=v_{i}^{R}, \quad \phi_{i+1}^{L}=\phi_{i}^{R}-M_{i}^{R} / k_{R}, \\
& N_{i+1}^{L}=N_{i}^{R}, \quad M_{i+1}^{L}=M_{i}^{R}, \quad Q_{i+1}^{L}=Q_{i}^{R}
\end{aligned}
$$

where $k_{T}, k_{R}=$ the spring constant of the joint for translation and rotation, respectively.

\section{Results and considerations}

Numerical computations for the aseismic reliability for the lifeline systems are conducted for the water supply pipeline system models in the assumption case and Yatsushiro city.

Figure 4 shows the water supply pipeline model in Yatsushiro city in which this area is expanded by $12 \mathrm{~km}$ (EW direction) $x 8 \mathrm{~km}$ (NS direction), and the pipeline system is modelled for the distribution lines of the diameter larger than $50 \mathrm{~mm}$ as 146 links, 138 nodes and 3 distribution basins. This area is divided into the mesh of $500 \mathrm{~m} \times 500 \mathrm{~m}$. The conditions of the ground in one mesh are assumed to be the same.

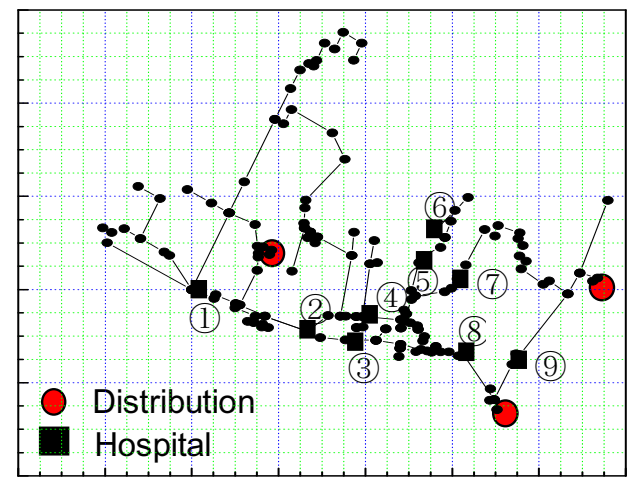

Figure 4: Water supply model of Yatsushiro city.

Table 1: $\quad$ Reliability of hospital lifeline.

\begin{tabular}{|c|c|c|}
\hline \multirow{2}{*}{ Hospital } & \multicolumn{2}{|c|}{ Reliability } \\
\cline { 2 - 3 } & I 5 & I 6 \\
\hline (1) $\mathrm{A}$ & 0.642 & 0.021 \\
\hline (2) $\mathrm{B}$ & 0.860 & 0.058 \\
\hline (3) $\mathrm{C}$ & 0.935 & 0.058 \\
\hline (4) $\mathrm{D}$ & 0.931 & 0.160 \\
\hline (5) $\mathrm{E}$ & 0.879 & 0.101 \\
\hline (6) $\mathrm{F}$ & 0.790 & 0.048 \\
\hline (7) $\mathrm{G}$ & 0.918 & 0.132 \\
\hline (8) $\mathrm{H}$ & 0.937 & 0.239 \\
\hline (9) $\mathrm{I}$ & 0 & 0 \\
\hline
\end{tabular}


Table 1 shows the reliabilities of nodes that are connected to the nine hospitals in Yatsushiro city for the cases of seismic intensity 5 and 6. In Table 2, the reliability means the water supply rate from the basin to the node. The average value of the reliability in the whole of Yatsushiro city is about 0.787 and 0.203 for seismic intensity 5 and 6 , respectively. In the case of seismic intensity 6 , the reliabilities of almost all of the hospitals are very low values. This suggests that another countermeasure is needed in addition to physical measures such as reinforcement of pipelines and ground improvement.

An example of the surface layers model of Kobe ground by 2D-FE is shown in Figure 5. This cross section of the width of $2,250 \mathrm{~m}$ and the depth of $20 \mathrm{~m}$ to $30 \mathrm{~m}$ are divided into 100 and 6 finite elements, respectively. The ground models rest on the rigid base rock surface and have absorbing (viscous) boundaries (A. B.) at both sides. The left and right sides of the diagrams are towards the mountain and sea sides, respectively. In the diagrams, $\mathrm{N}$ denotes the standard penetration test $\mathrm{N}$-value, which is converted to the initial soil stiffness (shear modulus) in the analysis. The geological parameters in Figure 5 are given as: A1 denotes the diluvial sandy gravel; A2 the alluvial sandy gravel; A3 the reclaimed soft soil; A5 the alluvial clay. The Kobe earthquake recorded at GL-32m in Port Island is used as input seismic acceleration waves. The NS and UD components of this input waves are impinged simultaneously upward from the horizontal base surface of the ground models with the maximum acceleration amplitude $A_{\max }$ of $0.1,1.0,5.4$ and $10.0 \mathrm{~m} / \mathrm{s}^{2}$.

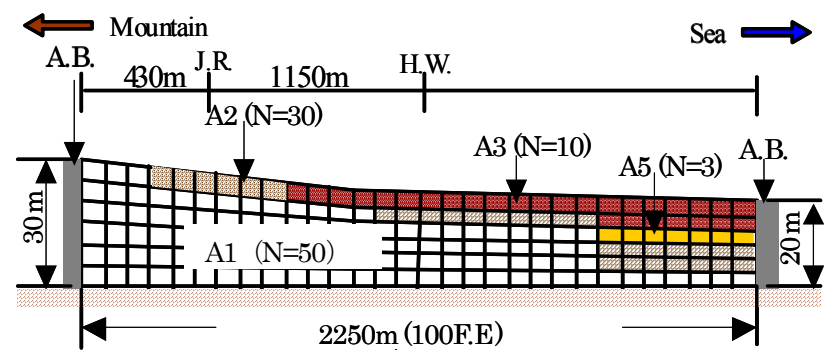

Figure 5: $\quad$ Example of surface ground layers model.

Figure 6 shows the distribution of the maximum ground strain along a cross section for four cases of the maximum acceleration amplitude $A_{\max }$. Large ground strain appears near the coastal areas, which may refer to heavily damaged areas of pipelines.

Numerical computations are carried out for the responses of axial and lateral displacements and internal forces of pipelines. The input ground displacement is assumed to be uniformly distributed over the pipeline length. The reference pipeline dimensions are shown in Table 2. The reference soil spring characteristic is assumed to be represented by the bi-linear coefficient of the subgrade reaction as shown in Figure 7. The GM-type joint, the resistance characteristics of which are shown in Figure 8, is used as the reference joint for 
segmented pipelines. The pipeline cases of the both ends fixed type and the cantilever type are evaluated while being subjected to lateral and longitudinal ground input, respectively.

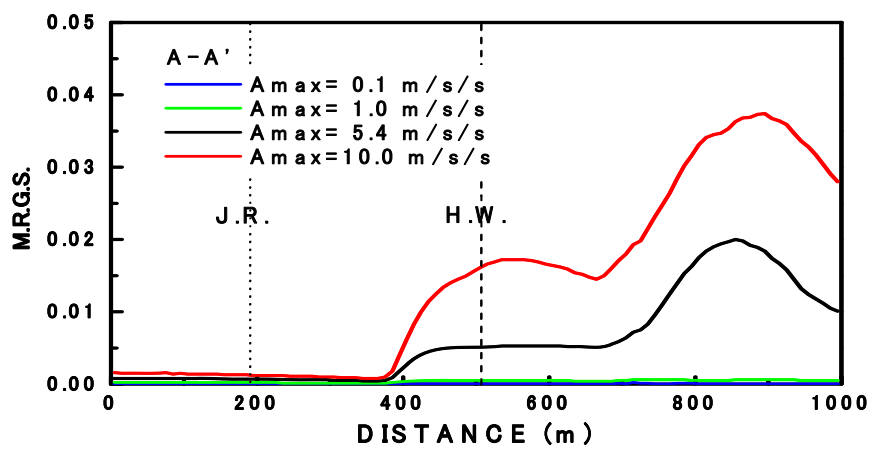

Figure 6: Maximum response ground strain near the surface (G.L.-2m).

Table 2: $\quad$ Reference pipe parameters.

\begin{tabular}{|c|c|}
\hline Physical items & Values (unit) \\
\hline Material of segment & Ductile cast iron \\
\hline Nominal diameter & $500(\mathrm{~mm})$ \\
\hline Thickness & $9.5(\mathrm{~mm})$ \\
\hline Total length & $100(\mathrm{~m})$ \\
\hline Young modulus & $1.57 \times 10^{8}\left(\mathrm{kN} / \mathrm{m}^{2}\right)$ \\
\hline Specific gravity & 7.15 \\
\hline Tensile strength & $3.92 \times 10^{5}\left(\mathrm{kN} / \mathrm{m}^{2}\right)$ \\
\hline Bending strength & $5.59 \times 10^{5}\left(\mathrm{kN} / \mathrm{m}^{2}\right)$ \\
\hline $\begin{array}{c}\text { Allowable joint } \\
\text { expansion }\end{array}$ & $50(\mathrm{~mm})$ \\
\hline $\begin{array}{c}\text { Allowable joint rot. } \\
\text { angle }\end{array}$ & $5($ degree $)$ \\
\hline
\end{tabular}

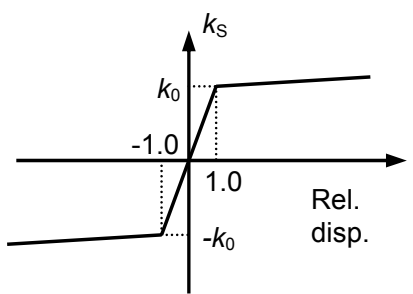

Figure 7: Characteristics of a soil spring. 
Figure 9(a) and (b) shows the distribution of the responses of displacement and the axial force of pipelines, respectively. Axial input soil displacement of $0.01 \mathrm{~m}$ per unit length $1 \mathrm{~m}$ is almost accumulated at the joint as the expansion displacement. Maximum axial force is $3200 \mathrm{kN}$, which means the maximum axial stress of about $58900 \mathrm{kn} / \mathrm{m}^{2}$ is below tensile strength, but maximum joint expansion is $58.6 \mathrm{~mm}$, larger than the allowable maximum of $50 \mathrm{~mm}$.

Bending moment $(\mathrm{kN} \cdot \mathrm{m})$

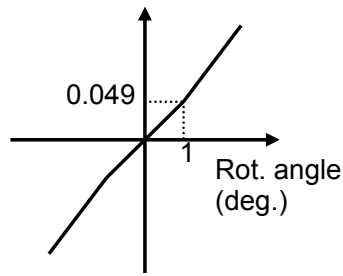

(a) Rotational joint spring

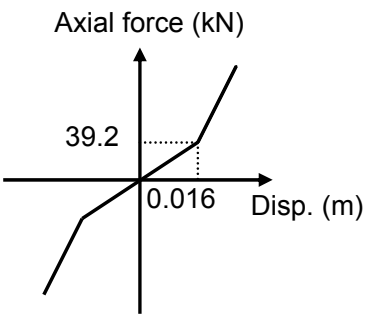

(b) Axial joint spring

Figure 8: Characteristics of the GM-type joint.

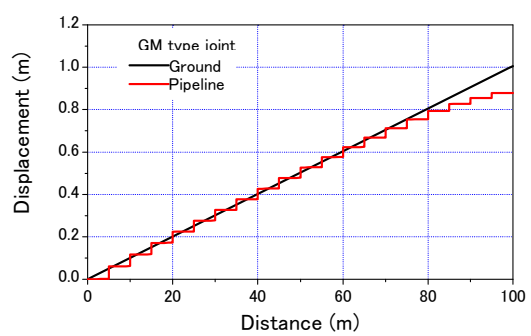

(a) Displacements

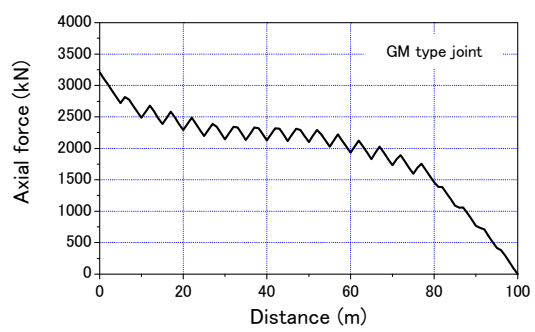

(b) Axial forces

Figure 9: Distribution of responses of pipelines.

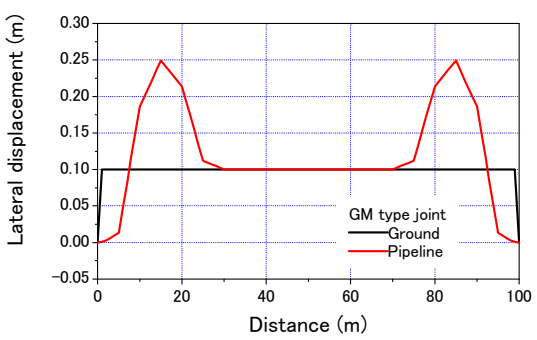

(a) Displacements

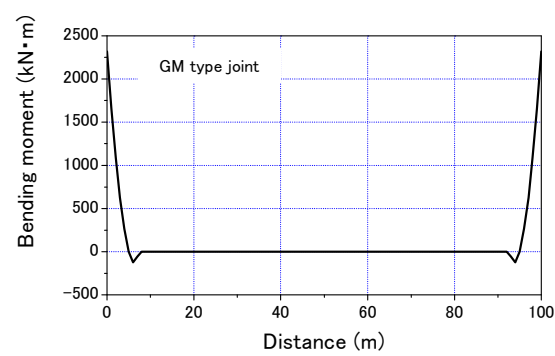

(b) Bending moments

Figure 10: Distribution of responses of pipelines. 
Figure 10(a) and (b) shows the distribution of the responses of lateral displacement and the bending moment of pipelines, respectively. The maximum lateral displacement of the pipeline is $0.25 \mathrm{~m}$ for $0.1 \mathrm{~m}$ input of soil. The maximum bending moment is $2300 \mathrm{kNm}$, which means the bending stress is about $160000 \mathrm{kN} / \mathrm{m}^{2}$ below tensile strength.

Figure 11(a) and (b) shows the same distributions of responses as Figure 10. In Figure 11 the responses in the case of liquefaction are shown in which the pipeline is subjected to $5 \mathrm{~m}$ lateral flow displacement of soil. Maximum lateral displacement of the pipeline is about $10 \mathrm{~m}$. The maximum joint rotational angle reaches about 19 degrees, which is larger than the allowable value of 5 degrees. This means breakage of the joint.

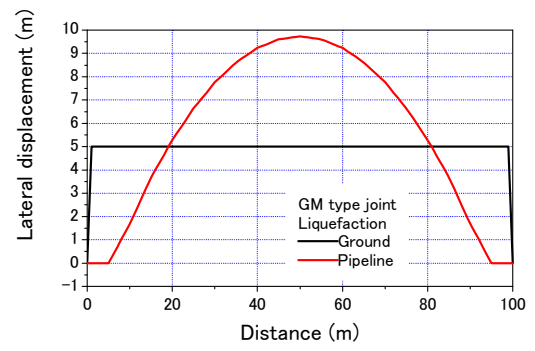

(a) Displacements

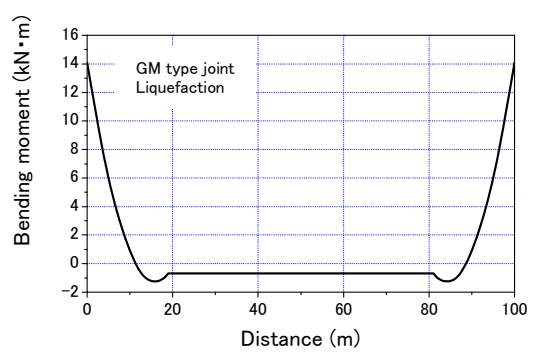

(b) Bending moments

Figure 11: Distribution of responses of pipelines.

\section{Conclusions}

In this study, the synthetic evaluation method for pipelines is proposed. The method consists of three different kinds of analyses, which are the seismic reliability analysis of the lifeline network system, seismic response analysis of surface ground layers and the pseudo static response analysis of pipelines. Some numerical computational examples of pipeline responses are investigated. The proposed method is effective in evaluating the situation of the whole network system in perspective and the detailed pipeline responses, including the response characteristics of surface ground layers.

\section{References}

[1] Akiyoshi, T., Fuchida, K. \& Maeda, S., An Estimation Method on Aseismic Investment for Lifeline Systems, Proc. of 10th Earthquake Engineering Symposium, Vol.3, pp.3187-3192, 1998 (in Japanese).

[2] Tamura, C. and Kawakami, H., Seismic risk analysis of underground lifeline system by use of Monte Carlo method, Proc. of JSCE, No.311, pp.37 48, 1981 (in Japanese).

[3] Japan Road Association, Specifications for highway bridges, Part V Earthquake -resistant design, JRA, 1996 (in Japanese). 
[4] Akiyoshi, T., Fuchida, K. \& Fang, H. L., “Absorbing boundary conditions for dynamic analysis of fluid-saturated porous media", Soil Dynamics and Earthquake Engineering, 1994, 13(4), 387-397.

[5] Akiyoshi, T., Fuchida, K. et al., A Nonlinear Seismic Response Analysis Method for Saturated Soil-Structure System with Absorbing Boundary, Int. J. Numeric. and Analytic. Methods in Geomaech., 20(5), pp.307-329, 1996.

[6] Iai, S. et al., Strain space plasticity model for cyclic mobility, Soils and Foundations, JSSMFE, Vol.32, No.2, pp.1-15, 1992.

[7] Akiyoshi, T., Fuchida, K. \& Shirinashihama, S., Local site effects of transient dynamic characteristics of irregularly layered grounds, Proc. Fourth Int. Conf. on Recent Advances in Geotech. Earthq. Eng. And Soil Dynamics, Paper No.3.11, 2001.

[8] Fuchida, K., Wang, L.R.L. \& Akiyoshi, T., Parametric Analysis of Buried Pipelines Subjected to Liquefied Ground Movements, Proc. of 5th U.S. National Conference on Earthquake Engineering, pp.959-968, 1994.

[9] Fuchida, K., Akiyoshi, T., Sun, X., Preventive effect of ground improvement on liquefaction and destructive deformation of pipelines, Proc. of Third China-Japan-US Trilateral Symposium on Lifeline Earthquake Engineering, pp.141-148, 1998. 